R. López Rodríguez, E. González Babarro, J. Campos Franco, M. R. Alende Sixto, A. González Quintela

Servicio de Medicina Interna. Complejo Hospitalario Universitario de Santiago de Compostela. Santiago de Compostela. A Coruña

1. Patel SR, Oleginski TP, Perruquet JL, Harrington TM. Pyomyositis: clinical features and predisposing conditions. J Rheumatol 1997; 24: 1734-38

2. Yoneda M, Oda K. Type 2 diabetes complicated by multiple pyomyositis. Intern Med 2003; 42: 174-77.

3. Christin L, Sarosi GA. Pyomyositis in North America: case reports and review. Clin Infect Dis 1992; 15: 668-77.

4. Merrien D, Hamidou M, Billaud E, Magadur G, Mussini JM, Raffi F. Bacterial pyomyositis. Analysis of 20 cases an review. Eur J Int Med 1995; 6: 229-34.

5. Gómez-Reino JJ, Aznar JJ, Pablos JL, Diaz-Gonzalez F, Laffon A Nontropical pyomyositis in adults. Semin Arthritis Rheum 1994; 23: 396-405.

6. Barros Alcalde P, López Rodríguez R, Lado Lado FL, Campos Franco J, Alende Sixto R, Rodríguez Constenla I. Piomiositis no tropical. An Med Interna (Madrid) 2004; 21: 567-8.

7. Bickels J, Ben-Sira L, Kessler A, Wientroub S. Primary pyomyositis. J Bone Joint Surg 2002; 84: 2277-85.

8. Jiménez-Mejías ME, Lozano de León F, Alfaro-García MJ, et al. Piomiositis por Staphylococcus aureus. Med Clin (Barc) 1992; 99: 201-5.

9. Cambronero JA, Yus S, Rogero S, López J, Jiménez J. Infecciones graves de partes blandas. Enf Infecc Microbiol Clin 1989; 7 (Supl. 1): 1622.

10. Soler R, Rodríguez E, Aguilera C, Fernández R. Magnetic resonance imaging of pyomyositis in 43 cases. Eur J Radiol 2000; 35: 59-64.

\section{Calcificaciones cerebrales en hipoparatiroidismo idiopático crónico}

\section{Sr. Director:}

Las calcificaciones cerebrales que afectan a ganglios basales se detectan en 0,68-0,93\% de tomografías computerizadas (TC) craneales $(1,2)$, habiéndose descrito múltiples patologías causantes o asociadas (3). Una de las etiologías a considerar son los trastornos del metabolismo fosfocálcico, principalmente hipoparatiroidismo (idiopático, postoperatorio) y seudohipoparatiroidismo; aunque también se han descrito en hiperparatiroidismo (primario, secundario a insuficiencia renal crónica), y en hipovitaminosis D sin hipoparatiroidismo $(4,5)$.

Se presenta un caso de hipoparatiroidismo idiopático crónico con calcificaciones cerebrales.

Varón, 71 años, con antecedentes patológicos de hipertensión arterial en tratamiento con torasemida $(2,5 \mathrm{mg} /$ día $)$ y trastorno ansioso-depresivo en tratamiento con bromazepam (3 $\mathrm{mg} /$ día). Diagnosticado hacía 20 años de hipoparatiroidismo idiopático, en tratamiento con suplementos de calcio (3 comprimidos/día) y vitamina D (1 comprimido/día); en los últimos 7 años el paciente había reducido la dosis de suplemento cálcico a 1 comprimido diario, sin toma de vitamina $\mathrm{D}$, y sin controles médicos por esta patología. Acudió a Urgencias por cuadro agudo de ansiedad, parestesias peribucales, en manos y pies y contractura palmoplantar. En exploración física, el paciente estaba vigil, tembloroso, sudoroso, con espasmo carpopedal y mioclonías. En analítica, destacaba hipocalcemia severa (Ca $3,8 \mathrm{mg} / \mathrm{dl})$, hiperfosfatemia (P 5,7 mg/dl), hipomagnesemia (Mg 1,79 mg/dl; normal:1,9-2,5 $\mathrm{mg} / \mathrm{dl}$ ), y rabdomiólisis moderada (CK 1.125 UI/l, CK-MB 23 UI/l). Hemograma, coagulación, gasometría arterial, y orina eran normales. Hormona paratiroidea (PTH) intacta baja $(2,1 \mathrm{pg} / \mathrm{ml}$; normal: $10-65 \mathrm{pg} / \mathrm{ml}$ ). Los valores de metabolitos de la vitamina D (25 OH y $1,25 \mathrm{OH}_{2}$ ), hormonas tiroideas (TSH y T4libre), corticotropina (ACTH) y cortisol fueron normales. En ECG, ritmo sinusal a $90 \mathrm{lpm}$, eje QRS normal, intervalo PR normal y QT corregido alargado $(530 \mathrm{~ms})$. En radiografía de tórax, signos degenerativos vertebrales, sin otros hallazgos patológicos. En TC craneal (Fig. 1) se detectan calcificaciones bilaterales y simétricas en núcleos dentados cerebelosos (A), ganglios basales (globus pallidus y cabeza del núcleo caudado), cápsula interna, tálamo (B y C), y en sustancia blanca de corona radiata (D).

Se pautaron suplementos orales de calcio (2 g/día de Ca elemento), y vitamina D (calcitriol: $1 \mathrm{mg} /$ día), con desaparición de tetania y normalización de $\mathrm{CK}$. Los niveles de $\mathrm{Ca}$ al alta $\left(5^{\circ}\right.$ día de ingreso) eran 7,3 mg/dl. En control clínico-analítico ambulatorio a las 2 semanas el paciente estaba asintomático, con $\mathrm{Ca} 8,1$ $\mathrm{mg} / \mathrm{dl}$.

La asociación clínico-radiológica de hipoparatiroidismo crónico y calcificaciones bilaterales simétricas en ganglios basales se describió inicialmente en 1939 (6), y está bien documentada (7), recomendándose la evaluación de la función paratiroidea y metabolismo fosfocálcico en todos los pacientes a los que incidentalmente se detecten estas calcificaciones en pruebas de neuroimagen con TC. La aparición de calcificaciones cerebrales en el hipoparatiroidismo se ha relacionado con la duración de la hipo-
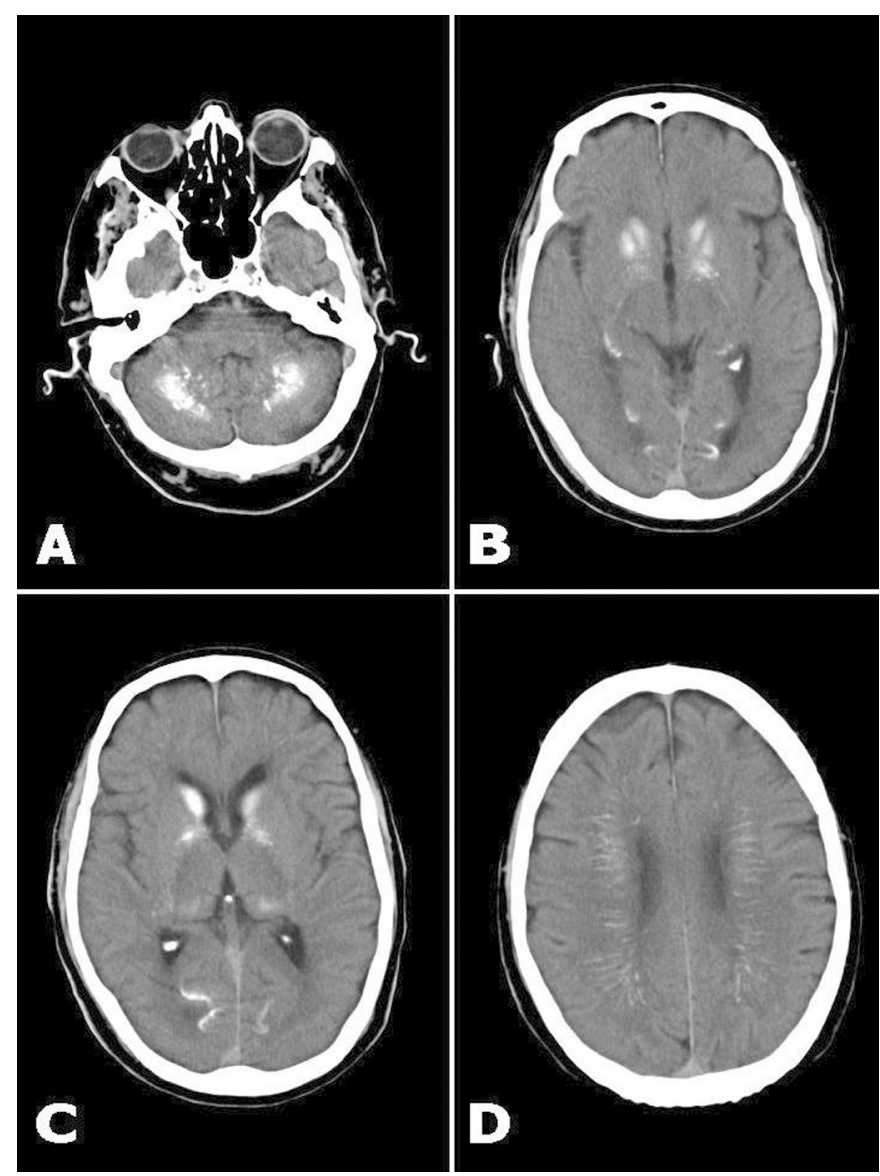

Fig. 1. En TC craneal se detectan calcificaciones bilaterales y simétricas en núcleos dentados cerebelosos (A), ganglios basales (globus pallidus y cabeza del núcleo caudado), cápsula interna, tálamo (B y C), y en sustancia blanca de corona radiata (D). 
calcemia e hiperfosfatemia, además de otros factores (anoxia cerebral, insuficiencia vascular) (8). En la enfermedad de Fahr, consistente en calcificaciones en ganglios basales y núcleos dentados, de carácter familiar (herencia autonómica dominante), clínica de retraso mental, epilepsia, atetosis y parálisis espástica, no hay hipocalcemia ni hipoparatiroidismo (9). Las calcificaciones cerebrales en el hipoparatiroidismo se han relacionado con crisis comiciales, síntomas extrapiramidales (coreoatetosis y parkinsonismo), cerebelosos (ataxia, disartria) y trastornos neuropsiquíatricos (ansiedad, depresión, labilidad emocional, retraso mental, demencia, psicosis) $(10,11)$, siendo difícil establecer en cada caso si estas manifestaciones neurológicas se deben a las alteraciones electrolíticas del hipoparatiroidismo (hipocalcemia, hiperfosfatemia, hipomagnesemia) o a la afectación estructural secundaria a las calcificaciones cerebrales, como en la enfermedad de Fahr.

\section{E. Crespo Valadés, A. Blanco Jarava, M. Malmierca Corral}

\section{Servicio de Medicina Interna. Hospital Virgen de la Salud. Toledo}

1. Sanchetee P, Venkataraman S, Mohan C, Shetty DD, Iyengar G. Basal ganglia calcification. J Assoc Physicians India 1999; 47: 507-9.

2. Tedrus GM, Fonseca LC, Nogueira E Jr. Basal ganglia calcification on computed tomography: clinical characteristics in 25 patients. Arq Neuropsiquiatr 2006; 64: 104-7.

3. Piñol-Ripoll G, Mauri-Llerda JA, de la Puerta Martínez-Miró I, PérezLázaro C, Beltrán-Marín I, López del Val LJ, et al. Diagnóstico diferencial de las calcificaciones intracraneales. Rev Neurol 2005; 41: 151-5.

4. Calvo Romero JM. Calcificaciones cerebrales múltiples asintomáticas y el eje vitamina D-hormona paratiroidea. Rev Neurol 2001; 32: 1198-9.

5. Jover-Díaz F, Pomares F, Matarranz M. Calcificaciones intracraneales extensas en el hipoparatiroidismo crónico. Rev Neurol 2006; 42: 61-2.

6. Eaton LM, Camp JD, Love JG. Symmetric cerebral calcification, particularly of the basal ganglia, demonstrable roentgenographically; calcification of the finer cerebral blood vessels. Arch Neurol Psychiatry 1939; 41: 921-42.

7. Sachs CH, Sjöberg HE, Ericson K. Basal ganglia calcifications on CT: Relation to hypoparathyroidism. Neurology 1982; 32: 779-82.

8. Fujita T. Mechanism of intracerebral calcification in hypoparathyroidism. Clin Calcium 2004; 14: 55-7.

9. Cartier L, Passig C, Gormaz A, Lopez J. Cambios neuropsicológicos y neurofisiológicos en la enfermedad de Fahr. Rev Med Chile 2002; 130: $1283-90$

10. Forstl H, Krumm B, Eden S, Kohlmyer K. Neurological disorders in 166 patients with basal ganglia calcification: a statistical evaluation. J Neurol 1992; 239: 36-8.

11. Kowdley KV, Coull BM, Orwoll ES. Cognitive impairment and intracranial calcification in chronic hypoparathyroidism. Am J Med Scien 1999; 317: 273-9.

\section{Hemoptisis y dorsalgia}

\section{Sr. Director:}

La espondilitis tuberculosa o "mal de Pott" es un proceso conocido desde la antigüedad (1), pero excepcional en la actualidad (2). Describimos a continuación un caso de esta modalidad de tuberculosis.

Varón de 21 años, natural de Marruecos y residentes en España desde hace varios meses, con nefrolitiasis como único antecedente de interés. Ingresó por haber presentado durante las horas previas tos con abundante expectoración hemoptoica y disnea. En los días previos había tenido tos con expectoración purulenta, sin fiebre, y a lo largo de los meses previos había notado dolor de progresiva intensidad en la región dorsal. A la exploración presentaba un buen aspecto general y unas constantes vitales normales, la auscultación pulmonar puso de manifiesto una disminución del murmullo vesicular en el hemitórax derecho, mientras que el resto de exploración fue normal.

Los análisis de sangre, incluidos hemograma, bioquímica completa, coagulación y proteinograma fueron normales. La gasometría arterial proporcionó los siguientes resultados: $\mathrm{pH}$ $7,39, \mathrm{pCO}_{2} 39 \mathrm{~mm} \mathrm{Hg}$ y pO, $60 \mathrm{~mm} \mathrm{Hg}$. El análisis de orina fue normal. El electrocardiograma no mostró alteraciones. En las radiografías de tórax se observaron un infiltrado cavitado en el lóbulo superior derecho y una condensación en el lóbulo inferior izquierdo. La fibrobroncoscopia mostró la existencia de sangre en todo el árbol bronquial derecho, procedente del bronquio del lóbulo superior derecho; la tinción de auramina-rodamina del aspirado bronquial fue positiva. A la vista de dicho resultado se inició tratamiento con isoniazida, rifampicina y pirazinamida.

Para completar el estudio de los infiltrados pulmonares y evaluar el dolor dorsal se efectuó una tomografía computarizada y una resonancia magnética del tórax y la columna vertebral que pusieron de manifiesto la existencia de una espóndilodiscitis dorsal D9-D10 y

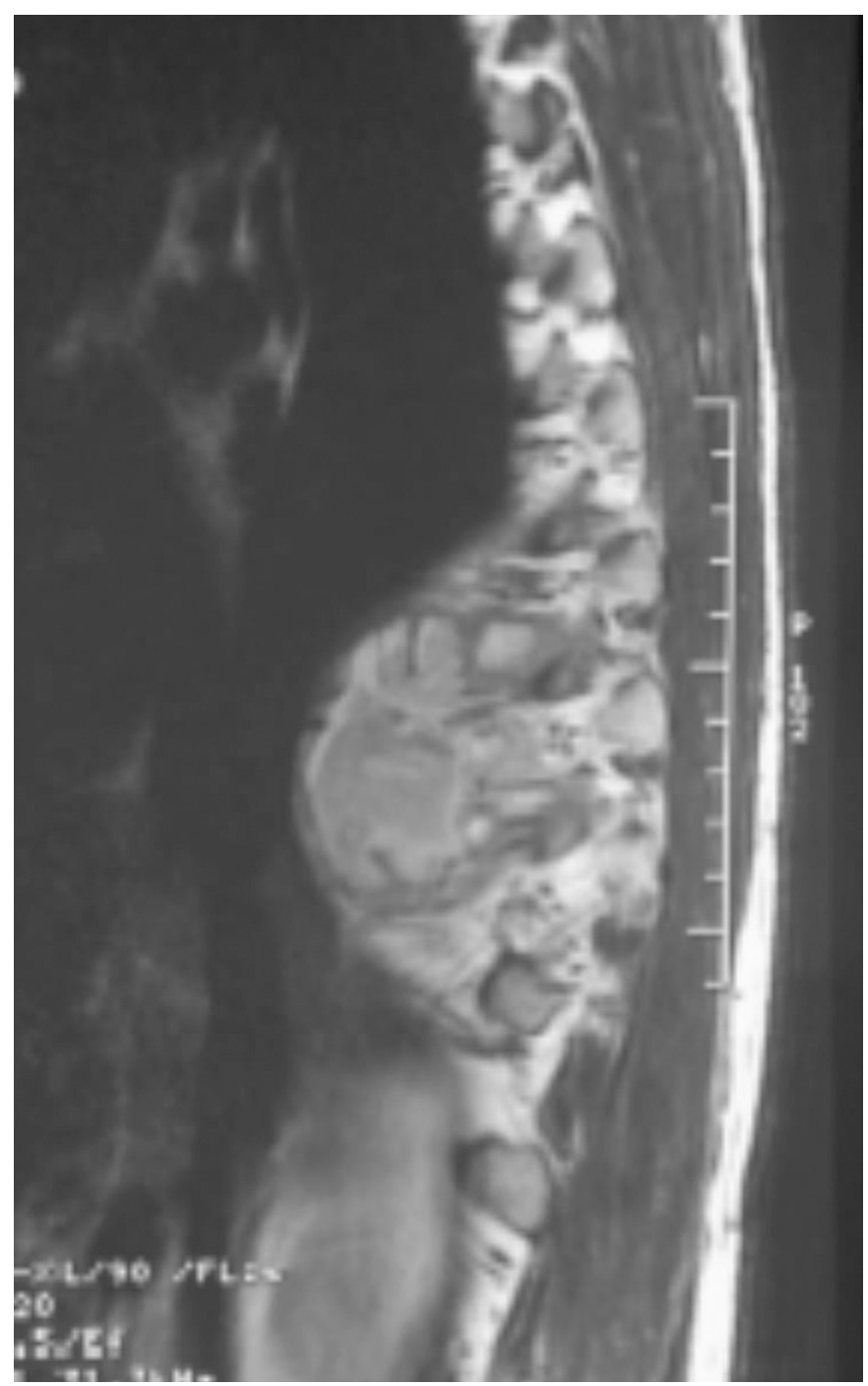

Figs. 1. Resonancia magnética de la columna vertebral (proyección sagital) que muestra una espondilodiscitis dorsal D9-D10 y D10D11, junto con un gran absceso prevertebral. 